\title{
Inaugural issue of Biomaterials and Tissue Engineering Bulletin
}

\author{
Michael R Hamblin ${ }^{1,2,3, *}$, Alexandru Mihai Grumezescu ${ }^{4, *}$
}

1 Wellman Center for Photomedicine, Massachusetts General Hospital, Boston MA, 02114, USA

2 Department of Dermatology, Harvard Medical School, Boston MA, 02115, USA

3 Harvard-MIT Division of Health Sciences and Technology, Cambridge, MA, 02139, USA

4 Department of Science and Engineering of Oxide Materials and Nanomaterials, Faculty of Applied Chemistry and Materials Science, University Politehnica of Bucharest, 1-7 Polizu Street, 011061 Bucharest, Romania

*corresponding author e-mail address: hamblin@helix.mgh.harvard.edu alexandru.grumezescu@upb.ro

\section{EDITORIAL}

It is our pleasure and honor to announce the launch of the inaugural issue of Biomaterials and Tissue Engineering Bulletin, which is supported by AMG Transcend (Romania).

Biomaterials and Tissue Engineering Bulletin is an academic, interdisciplinary, open-access journal, which provides the opportunity for scientists and researchers to share the latest and most advanced results regarding the current development of innovative biomaterials and new trends in tissue engineering. Biomaterials play a crucial role in the fields of life science, material engineering, regenerative medicine and modern healthcare practice. Biomaterials are nonviable complex systems that interact with living organisms, in order to maintain, restore and improve their biological functions. Likewise, tissue engineering employs concepts from the above-mentioned fields and takes advantage of the tremendous impact that stem cells and progenitor cells have had in biomedicine, and are expected to have in the new era of personalized medicine. Tissue engineering aims to develop biological substitutes that improve or restore tissue and organ function to treat disease and even to extend lifespan.

The scope of Biomaterials and Tissue Engineering Bulletin is to publish original experimental papers and up-to-date review articles concerning the development and physical, chemical and biological characterization of biomaterials, as well as discussion of their present and future applications in biomedical research. Its scope will cover in vitro and in vivo preclinical studies and clinical reports. Submitted original papers should make a significant contribution in terms of understanding, development and future application of biomaterials and tissue engineering to biomedicine and health-care practice.

Biomaterials and Tissue Engineering Bulletin covers (but is not limited to) key aspects of the following disciplines: synthesis, characterization and biomedical potential of metallic, ceramic, polymeric, composites and hybrid biomaterials; physical, chemical, biological, pharmaceutical and toxicological features of biomaterials; studies regarding the interactions between biomaterials and biological systems; design of biomaterials for modern diagnosis and therapeutic clinical practice (bioimaging, biosensing, biotherapy); drug delivery, protein delivery, gene delivery, prodrugs, molecular biopharmaceutics; design, development, refinement and biomedical impact of tissue scaffolds regarding hard and soft tissue engineering; promoting the concept of personalized treatment for different types of pathological conditions in the evolutionary context of modern medicine; advanced and innovative trends in health-care practice; all aspects of tissue engineering; organ fabrication; tissue printing; cellseeded matrices; clinical outcomes of engineered tissue implantation; cell-based therapies.

The publication costs of Biomaterials and Tissue Engineering Bulletin are covered by the AMG Transcend (Romania). Authors are not requested to pay any article-processing fees.

We cordially invite colleagues throughout the world, who are working in the fields of biomaterials and tissue engineering to share their most recent research and ideas through Biomaterials and Tissue Engineering Bulletin and help us advance outstanding knowledge in this dynamic field. 